Comparison of patient characteristics, clinical management, infectious specialist consultation, and outcome in men and women with methicillin-sensitive Staphylococcus aureus bacteremia : a propensity-score adjusted retrospective study

Forsblom, E.

2018-12

Forsblom , E , Kakriainen , A , Ruotsalainen , E \& Järvinen , A 2018 , ' Comparison of patient characteristics, clinical management, infectious specialist consultation, and outcome in men and women with methicillin-sensitive Staphylococcus aureus bacteremia : a propensity-score adjusted retrospective study ' , Infection , vol. 46 , no. 6 , pp. 837-845 . https://doi.org/10.1007/s15010-018-1216-3

http://hdl.handle.net/10138/276969

https://doi.org/10.1007/s15010-018-1216-3

publishedVersion

Downloaded from Helda, University of Helsinki institutional repository.

This is an electronic reprint of the original article.

This reprint may differ from the original in pagination and typographic detail.

Please cite the original version. 


\title{
Comparison of patient characteristics, clinical management, infectious specialist consultation, and outcome in men and women with methicillin-sensitive Staphylococcus aureus bacteremia: a propensity-score adjusted retrospective study
}

\author{
E. Forsblom ${ }^{1}$ - A. Kakriainen ${ }^{1}$ - E. Ruotsalainen ${ }^{1} \cdot$ A. Järvinen ${ }^{1}$
}

Received: 9 May 2018 / Accepted: 5 September 2018 / Published online: 7 September 2018

(c) Springer-Verlag GmbH Germany, part of Springer Nature 2018

\begin{abstract}
Background Sex-related treatment inequalities are suggested to explain outcome differences between men and women in Staphylococcus aureus bacteremia (SAB). We compared patient characteristics, clinical management, infectious specialist consultation (ISC) and outcome in men and women with SAB.

Methods Multicenter retrospective study of methicillin-sensitive (MS-) SAB patients categorized according to sex and ISC consultation provided within 7 days of diagnosis.

Results Altogether 617 SAB patients were included in the analysis: $62 \%$ males and 38\% females. Male sex was associated less often to nosocomial bacteremia (OR $0.69,95 \%$ CI $0.50-0.96, p=0.029)$ and more often to alcoholism (OR 2.25, 95\% CI 1.31-3.87, $p=0.003)$. No sex-related differences were seen in basic or immunologic laboratory tests, illness severity, intensive care unit treatment or thromboembolic events. ISC was provided to most patients (94\%) irrespective of sex. No differences were seen in clinical management of men or women: Transthoracic or -esophageal echocardiography (61\% vs. $65 \%$ ), deep infection (77\% vs. $72 \%)$, infection removal (30\% vs. $27 \%)$ and anti-staphylococcal antibiotics as first-line treatment $(54 \%$ vs. $51 \%$ ). However, male sex was connected to more frequent adjunctive rifampicin treatment ( $52 \%$ vs. $41 \%, p=0.025)$. No difference in 28 - or 90 -day mortality ( $13 \%$ vs. $13 \%$ and $18 \%$ vs. $20 \%$ ) or SAB relapse ( $0 \%$ vs. $1 \%$ ) was observed between men and women. Propensity-score adjusted Cox proportional analysis gave no connection of sex to mortality within 90 days. Conclusion Patient characteristics, clinical management, ISC guidance, bacteremia relapse, and outcome did not differ in men and women with MS-SAB.
\end{abstract}

Keywords S. aureus bacteremia $\cdot$ Infectious specialist consultation $\cdot$ Deep infection foci $\cdot$ Prognosis $\cdot$ Sex

\section{Introduction}

Staphylococcus aureus bacteremia (SAB) is associated with considerable morbidity and mortality [1]. Prognosis of SAB is influenced by age and comorbidity [2], severe sepsis [3], endocarditis [4] and methicillin-resistance [5]. However,

E. Forsblom and A. Kakriainen equal contribution.

E. Forsblom

erik.forsblom@helsinki.fi

1 Division of Infectious Diseases, Department of Medicine, Inflammation Center, Helsinki University Central Hospital, Aurora Hospital, University of Helsinki, Nordenskiöldinkatu 26, Building 3, P.O. Box 348, 00029 HUS Helsinki, Finland infectious disease specialist consultation (ISC) improves prognosis of SAB [6]. ISC as a mandatory practice in $\mathrm{SAB}$ is advocated by ever more clinicians $[7,8]$.

The impact of sex on prognosis in severe infections has received much attention. However, reports are conflicting, with female sex connecting to excess mortality in bacteremia [9], whereas sepsis survival studies present poorer outcome in males [10] or no sex-related prognostic differences [11].

Female sex has repeatedly been associated to poorer prognosis in SAB [12-17]. A recent large Danish population-based study confirmed that women had significantly higher mortality in SAB and suggested that female sex should be considered in risk stratification [13]. The connection of female sex to poor outcome in severe infections has been speculated to be due to sex-related dimorphism 
[18] including female sex steroids [19] enhancing immune response with potential subsequent impact on outcome. Studies on healthcare-seeking behavior speculate that women receive antibiotic prescriptions more often which may delay hospital arrival and influence prognosis [20]. Furthermore, studies on sepsis report care inequality for severely ill female patients with delayed empiric antimicrobials [21], less access to mechanical ventilation, hemodialysis [22] or life-supporting treatments [23]. However, prospective studies [1, 24] and ISC guided management reports $[4,6-8]$ have not reported any sex-related outcome disadvantage in SAB. These contradicting results were evaluated in a recent editorial [25] and explained by limited data on clinical management including incomplete reporting of ISC, lack of knowledge on infection acquisition and inclusion of poly-microbial infections or non-adjustment for co-morbidities [12-17].

The objective of the present study was to compare patient characteristics, clinical management, infectious specialist consultation, bacteremia relapse and outcome in men and women with methicillin-sensitive (MS-) SAB. Inclusion of MS-SAB only enabled each patients to receive proper nondelayed antibiotics from the first day of SAB and thus reducing empirical antibiotic influence.

\section{Materials and methods}

\section{Study population}

This was a retrospective multicenter study with 90 days follow-up. All adult patients with blood culture positive for $S$. aureus from five university and seven central hospitals in Finland during January to May 1999 and January 2000 to August 2002 were enrolled. Furthermore, as an extension, each adult patient with positive blood culture for $S$. aureus from Helsinki University Central Hospital in Finland in 2006-2007 was included. Bacteremia due to methicillinresistance (MRSA) was omitted. Two time-periods were included to account for temporary differences in treatment management, personnel practices or other factors difficult to control for studies. The median time-period between blood culture sampling and clinical awareness of $S$. aureus as a causative pathogen was 3 days. Data documentation included: sex, age, co-morbid diseases, illness severity, deep infection identification and eradication and administration route and length of antibiotic therapy and any ISC. Both basic laboratory tests and tests for immunological status were recorded repeatedly. Final statistical analyses were performed by excluding patients who deceased within 3 days to account for the possibility of death before performed ISC. The primary end-point was mortality at 28 and 90 days and secondary end-point defervescence time, SAB relapse and hospitalization duration. No differences in the deep infection foci, antimicrobial treatment or underlying characteristics were observed in patient groups from the two different inclusion periods.

\section{Definitions}

McCabe's criteria were applied for classification of comorbid diseases [26]. ISC within 7 days of S. aureus bacteremia diagnosis were recorded. Formal ISC was defined as a bedside consultation by the infectious specialist with review of patient records, physical investigation and written directives. Informal ISC was defined as a telephone consultation (or other informal consultation) where the treating physician documented into the patient records directives given by the ISC [27].

\section{Statistical analyses}

Categorical variables were compared with Pearson's $\chi^{2}$ test and non-categorical variables with Mann-Whitney $U$ test. Univariate parameters with $p \leq 0.1$ were accepted for further analyses. The independent prognostic impact of sex was estimated by propensity-score adjusted Cox proportional regression analysis. First, variables with significant statistical connection to sex were identified and interpreted as relevant. Second, a propensity-score adjusted Cox proportional regression analysis was performed to estimate prognostic parameters of 90-days outcome. All tests were two-tailed and $p<0.05$ regarded significant. Analyses were done with SPSS 12.0 (SPSS Inc. Chicago IL, USA).

\section{Results}

\section{Patient characteristics and illness severity}

A total of $617 \mathrm{SAB}$ patients were included. Altogether, $384(62 \%)$ were men aged $(56.0 \pm 17$ years, mean \pm SD) and $233(38 \%)$ women $(58.3 \pm 18$ years, mean \pm SD). Men, compared to women, had less nosocomial bacteremia (OR $0.69, p=0.029)$ and more alcoholism (OR 2.25, $p=0.003)$, whereas no significant differences were observed in hospitalization preceding $\mathrm{SAB}$ or in other underlying conditions (Table 1). No significant differences were seen between sex and PITT bacteremia scores (mean or total scores $\geq 3$ ), severe sepsis, intensive care unit or thromboembolic events (Table 1).

\section{Laboratory parameters}

Basic laboratory parameters were recorded for each patient and teichoic acid antibody and antistaphylolysin for 
Table 1 Patient demographics, underlying conditions and severity of illness in 617 patients with methicillinsensitive $S$. aureus bacteremia stratified according to sex

\begin{tabular}{|c|c|c|c|c|}
\hline Patient characteristics & $\begin{array}{l}\text { Women } \\
233 \text { (38) }\end{array}$ & $\begin{array}{l}\text { Men } \\
384 \text { (62) }\end{array}$ & OR $(95 \% \mathrm{CI})$ & $p$ value \\
\hline \multicolumn{5}{|l|}{ Demographics } \\
\hline Age $>60$ years & $119(51)$ & $167(43)$ & $0.74(0.53-1.02)$ & NS \\
\hline Age, years $(\text { mean } \pm S D)^{a}$ & $58.25 \pm 18.47$ & $56.02 \pm 17.30$ & - & NS \\
\hline Nosocomial bacteremia & $137(59)$ & $191(50)$ & $0.69(0.50-0.96)$ & 0.029 \\
\hline Previous hospitalization $^{\mathrm{b}}$ & $131(56)$ & $207(54)$ & $0.91(0.66-1.27)$ & NS \\
\hline \multicolumn{5}{|l|}{ Underlying conditions } \\
\hline Healthy—nonfatal disease ${ }^{c}$ & $173(74)$ & $263(68)$ & $0.75(0.52-1.09)$ & NS \\
\hline Ultimately_rapidly fatal disease ${ }^{c}$ & $60(26)$ & $121(32)$ & $1.33(0.92-1.91)$ & NS \\
\hline Coronary heart disease & $54(23)$ & $91(24)$ & $1.03(0.70-1.51)$ & NS \\
\hline Chronic pulmonary disease & $42(18)$ & $64(17)$ & $0.91(0.60-1.40)$ & NS \\
\hline Diabetes mellitus & $35(15)$ & $73(19)$ & $1.36(0.86-2.15)$ & NS \\
\hline Chronic renal failure & $26(11)$ & $57(15)$ & $1.39(0.85-2.28)$ & NS \\
\hline Chronic alcoholism & $19(8)$ & $64(17)$ & $2.25(1.31-3.87)$ & 0.003 \\
\hline HIV infection & $2(1)$ & $9(2)$ & $2.78(0.60-12.9)$ & NS \\
\hline Corticosteroids $^{\mathrm{d}}$ & $22(9)$ & $31(8)$ & $0.84(0.48-1.49)$ & NS \\
\hline \multicolumn{5}{|l|}{ Malignancy } \\
\hline Hematological & $16(7)$ & $23(6)$ & $0.86(0.45-1.67)$ & NS \\
\hline Non-hematological & $28(12)$ & $38(10)$ & $0.80(0.48-1.35)$ & NS \\
\hline \multicolumn{5}{|l|}{ Severity of illness } \\
\hline Severe sepsis ${ }^{f}$ & $17(7)$ & $39(10)$ & $1.44(0.79-2.60)$ & NS \\
\hline PITT score $\geq 3$ & $21(9)$ & $44(11)$ & $1.29(0.75-2.24)$ & NS \\
\hline PITT score $(\text { mean } \pm \mathrm{SD})^{\mathrm{a}}$ & $0.65 \pm 1.45$ & $0.89 \pm 1.82$ & - & NS \\
\hline ICU treatment ${ }^{\mathrm{e}}$ & $44(19)$ & $80(21)$ & $0.75-1.70)$ & NS \\
\hline Non-invasive ventilation & $9(4)$ & $22(6)$ & $0.68-3.34)$ & NS \\
\hline Inotrope support & $9(4)$ & $23(6)$ & $0.72-3.49)$ & NS \\
\hline Acute dialysis & $7(3)$ & $15(4)$ & $1.31(0.53-3.27)$ & NS \\
\hline Thromboembolic events $^{\mathrm{e}}$ & $31(13)$ & $46(12)$ & $0.87(0.53-1.45)$ & NS \\
\hline \multicolumn{5}{|l|}{ Basic parameters ${ }^{\mathrm{e}}$} \\
\hline \multicolumn{5}{|l|}{ C-reactive protein } \\
\hline At blood cultures & $175 \pm 114$ & $175 \pm 107$ & - & NS \\
\hline At day 3 & $136 \pm 79$ & $132 \pm 83$ & - & NS \\
\hline At day 7 & $73 \pm 64$ & $72 \pm 55$ & - & NS \\
\hline Leucocyte count & $13 \pm 5.6$ & $14 \pm 15$ & - & NS \\
\hline Blood glucose & $8.4 \pm 4.5$ & $8.4 \pm 5.1$ & - & NS \\
\hline Albumin (liver) & $26 \pm 5.8$ & $26 \pm 7.5$ & - & NS \\
\hline Alanine aminotransferase & $70 \pm 151$ & $88 \pm 361$ & - & NS \\
\hline Activated partial thromboplastin time & $36 \pm 4$ & $39 \pm 6$ & - & NS \\
\hline
\end{tabular}

Values are $\mathrm{n}(\%)$ and odds ratios (ORs) with 95\% confidence intervals (CI) or mean \pm standard deviation (SD). Comparisons with Mann-Whitney $U$ test

$N S$ non-significant

a Mann-Whitney $U$ test

${ }^{\mathrm{b}}$ Within 2 months prior to SAB

${ }^{\mathrm{c}}$ McCabe's classification [26]

${ }^{\mathrm{d}}$ Dose $\geq 10 \mathrm{mg} /$ day $\geq 1$ month

${ }^{\mathrm{e}}$ At blood culture collection one-third of patients. Parameters relevant for coagulation and immunology were retrieved for only part of patients. Basic parameters of C-reactive protein, leucocyte count, blood glucose, albumin, alanine aminotransferase and activated partial thromboplastin were measured at daily basis during the first 7 days and thereafter once a week. Parameters for 
coagulation, i.e., anti-thrombin III, fibrinogen, thrombin time, factor VIII and d-dimer $(N=42)$ were recorded at positive blood cultures, at days 7 and 90 . Immunoglobulins A, M and $\mathrm{G}(N=22)$ and complement factors $\mathrm{C}-3, \mathrm{C}-4, \mathrm{Ch} 100 \mathrm{Cl}$ $(N=43)$ and interleucin-2 receptor $(N=10)$ were recorded once a week and teichoic acid antibody and antistaphylolysin $(N=256)$ at day 3 and 21. No sex-related difference for any laboratory test at any time-point was observed. The basic laboratory results are presented in Table 1 .

\section{Clinical management}

Altogether 94\% (582) of patients received ISC. Most patients had formal (bedside) ISC (84\%) whereas informal (telephone) ISC was provided to $62(10 \%)$ patients and only $35(6 \%)$ were managed without consultation. No significant difference with respect to sex were observed for presence or absence of ISC (Table 2). Echocardiography was provided to $63 \%$ of patients with no significant difference observed between men and women regarding transthoracic- (61\% vs. $65 \%)$ or trans-esophageal (18\% vs. $17 \%)$ examinations. Deep infection foci were identified in 75\% (464) and endocarditis in $15 \%$ (91) of patients without any sex differences (Table 2). Altogether 29\% (178) of patients underwent infection focus removal. No significant differences between men and women were noted regarding radiological or surgical infection focus eradication, infected joint lavage or heart valve replacement (Table 2). An intravenous antimicrobial agent effective in vitro against the $S$. aureus blood isolate was provided to each patient from the first day of positive blood culture. Most received anti-staphylococcal penicillin (53\%), whereas $37 \%$ had cephalosporin as a first-line treatment. No significant difference was observed regarding sex and standard antimicrobial or adjunctive fluoroquinolone antimicrobial therapy. However, rifampicin adjunctive therapy was provided more often to men compared to women (52\% vs. $41 \%$, $p=0.025$ ) (Table 2).

\section{Primary and secondary outcome}

The mortality was $13 \%$ at 28 days and $19 \%$ at 90 days with no significant difference observed between men and women (Table 2; Fig. 1). Furthermore, when analyzing the two time-periods (years 1999-2002 and 2006-2007) separately no differences in outcome were observed between men and women. Patients who deceased within 3 days $(N=12)$ were excluded from univariate and Cox proportional regression analysis to allow for death before the possibility of ISC. When comparing patients who died with 3 days no statistically significant differences were observed regarding sex: 8 (67\%) were men and 4 (33\%) women (OR 1.22). Mean time $( \pm$ SD) to defervescence and hospitalization were $6 \pm 9$ days and $35 \pm 30$ days with no sex difference (Table 2; Fig. 2).
Documentation regarding SAB relapse was retrieved for 430 patients and only $5(1 \%)$ had a SAB relapse within 90-day follow-up. No significant statistical difference was seen between sex and SAB relapse (Table 2). When comparing disease severity and treatment of deceased men and women no differences were observed (Table 3 ).

Parameters in univariate analysis connecting to 90-day mortality were: age $\geq 60$ years (OR $3.74, p<0.001$ ), healthy-nonfatal disease (OR $0.15, p<0.001$ ), intensive care unit treatment (OR 2.05, $p<0.01$ ), formal ISC (OR $0.48, p<0.01$ ), endocarditis (OR 2.12, $p<0.01$ ), pneumonia (OR 3.25, $p<0.001$ ) and adjunctive rifampicin therapy (OR $0.38, p<0.001$ ). The independent prognostic impact of sex was estimated by propensity-score adjusted Cox proportional regression. First, variables with significant statistical connection to sex were identified (Tables 1,2 ) and interpreted as relevant for the propensity-score assignment: (i) nosocomial bacteremia, (ii) alcoholism and (iii) rifampicin therapy. Second, a propensity-score adjusted Cox proportional regression analysis was performed for 90-day mortality: age $\geq 60$ years (HR $2.21, p<0.01$ ), healthy-nonfatal disease (HR $0.23, p<0.001$ ), intensive care unit treatment (HR $1.60, p<0.05$ ), formal ISC (HR $0.58, p<0.05$ ), endocarditis (HR 2.51, $p<0.001$ ), pneumonia (HR 2.42, $p<0.001$ ) and rifampicin therapy (HR 0.24, p < 0.001) (Table 4).

The connection of female sex to poor prognosis in SAB has been reported to be accentuated among patients with chronic pulmonary disease or malignancy [13]. As a further analysis we evaluated the connection of sex and outcome by including only patients with (i) chronic pulmonary disease, (ii) malignancy and (iii) patients with McCabe's classification for severe underlying conditions. However, mortalities at 28 and 90 days did not differ significantly between men and women with chronic pulmonary disease, malignancy or severe underlying illness according to McCabe's classification.

\section{Discussion}

The main observation was that patient characteristics, clinical management, ISC guidance, bacteremia relapse, and outcome did not differ in men and women with MS-SAB. In this setup, sex had no prognostic impact when accounting for prognostic parameters in propensity-score adjusted Cox proportional analysis.

The results in the present study are in contrast with previous nation- [12-14], region- [15] or single center [16] studies connecting female sex to poor outcome in SAB. We observed overall case fatalities of $13 \%$ and $19 \%$ at 28 and 90 days with no sex-related difference. Furthermore, when analyzing the two time-periods (years 1999-2002 and 2006-2007) separately no differences in outcome were 
Table 2 Clinical management with consultations, radiology, infection focus diagnostics and eradication, antimicrobial therapy, time to defervescence, hospitalization time and outcome in 617 patients with methicillin-sensitive Staphylococcus aureus bacteremia $(\mathrm{SAB})$ stratified according to sex

\begin{tabular}{|c|c|c|c|c|}
\hline Clinical management & $\begin{array}{l}\text { Women } \\
233(38)\end{array}$ & $\begin{array}{l}\text { Men } \\
384 \text { (62) }\end{array}$ & OR $(95 \% \mathrm{CI})$ & $p$ value \\
\hline \multicolumn{5}{|l|}{ Consultations and radiology } \\
\hline Formal (bedside) ISC ${ }^{\text {a }}$ & $194(83)$ & $326(85)$ & $1.13(0.72-1.76)$ & NS \\
\hline Informal (telephone) ISC ${ }^{a}$ & $25(11)$ & $37(10)$ & $0.89(0.52-1.52)$ & NS \\
\hline No ISC ${ }^{\mathrm{a}}$ & $14(6)$ & $21(5)$ & $0.91(0.45-1.82)$ & NS \\
\hline Transthoracic echocardiography & $153(65)$ & $236(61)$ & $0.79(0.56-1.11)$ & NS \\
\hline Trans-esophageal echocardiography & $41(17)$ & $71(18)$ & $1.06(0.69-1.62)$ & NS \\
\hline Whole-body computed tomography & $86(37)$ & $158(41)$ & $1.19(0.86-1.67)$ & NS \\
\hline \multicolumn{5}{|l|}{ Deep infection foci } \\
\hline Any deep focus & $168(72)$ & $296(77)$ & $1.30(0.90-1.89)$ & NS \\
\hline Pneumonia & $79(34)$ & $149(39)$ & $1.23(0.89-1.74)$ & NS \\
\hline Endocarditis & $33(14)$ & $58(15)$ & $1.08(0.68-1.71)$ & NS \\
\hline Osteomyelitis/septic arthritis & $72(31)$ & $140(36)$ & $1.29(0.91-1.82)$ & NS \\
\hline Deep-seated abscess & $86(37)$ & $152(40)$ & $1.12(0.80-1.57)$ & NS \\
\hline Foreign body foci & $40(17)$ & $51(13)$ & $0.74(0.47-1.16)$ & NS \\
\hline \multicolumn{5}{|l|}{ Infection removal $^{\mathrm{b}}$} \\
\hline Infected joint lavage & $6(3)$ & $7(2)$ & $0.70(0.23-2.12)$ & NS \\
\hline Heart valve replacement & $2(1)$ & $4(1)$ & $1.22(0.22-6.69)$ & NS \\
\hline Infected foreign body removal & $31(13)$ & $46(12)$ & $0.89(0.56-1.44)$ & NS \\
\hline Any infection eradication ${ }^{c}$ & $63(27)$ & $115(30)$ & $1.15(0.80-1.66)$ & NS \\
\hline \multicolumn{5}{|l|}{ Antimicrobial therapy } \\
\hline Vancomycin $^{\mathrm{d}}$ & $8(3)$ & $5(1)$ & $0.37(0.12-1.15)$ & NS \\
\hline Cephalosporin $^{\mathrm{d}}$ & $87(37)$ & $144(37)$ & $1.01(0.72-1.41)$ & NS \\
\hline Staphylococcal penicillin $^{\mathrm{d}}$ & $119(51)$ & $209(54)$ & $1.14(0.83-1.58)$ & NS \\
\hline Fluoroquinolone therapy ${ }^{\mathrm{e}}$ & $119(51)$ & $184(48)$ & $0.93(0.60-1.44)$ & NS \\
\hline Rifampicin therapy ${ }^{\mathrm{e}}$ & $96(41)$ & $194(52)$ & $1.46(1.05-2.02)$ & 0.025 \\
\hline Aminoglycoside ${ }^{\mathrm{e}}$ & $40(17)$ & $62(16)$ & $0.93(0.60-1.44)$ & NS \\
\hline \multicolumn{5}{|l|}{ Outcome } \\
\hline Defervescence (days) & $5 \pm 8$ & $7 \pm 10$ & - & NS \\
\hline Hospitalization (days) & $35 \pm 34$ & $34 \pm 29$ & - & NS \\
\hline SAB relapse within 90 days $^{\mathrm{f}}$ & 0 & $5(1)$ & - & NS \\
\hline Mortality within 28 days & $30(13)$ & $50(13)$ & $1.01(0.62-1.65)$ & NS \\
\hline Mortality within 90 days & $47(20)$ & $69(18)$ & $0.87(0.57-1.31)$ & NS \\
\hline
\end{tabular}

Values are expressed as $n(\%)$ and odds ratios (ORs) with 95\% confidence intervals (CI) or mean \pm standard deviation (SD) and comparisons with Mann-Whitney $U$ test

$N S$ non-significant

${ }^{a}$ Infectious specialist consultation

${ }^{\mathrm{b}}$ Data retrieved for 430 patients

${ }^{\mathrm{c}}$ Radiological or surgical

${ }^{\mathrm{d}}$ Standard therapy

${ }^{\mathrm{e}}$ Adjunctive therapy

${ }^{\mathrm{f}}$ Data for 430 patients observed between men and women. The mortality figures of the present study are considerably lower compared to 30 days case-fatality of $22-38 \%$ for males and $26-44 \%$ for females [12-16]. In the present study patients who deceased within 3 days were excluded from the final analyzes to allow for death before the possibility of ISC. This naturally might have influenced final results. However, only 12 patients $(67 \%$ men and 33\% women) deceased within the first 3 days and hence it is reasonable to assume that the exclusion of these patients has had only marginal impact on final results. We might have missed some more severe cases but the most likely explanation for the lower mortality is the meticulous localization, identification, and eradication of deep infection focus compared to previous studies $[13,15,16]$. Previous 
Figure 1

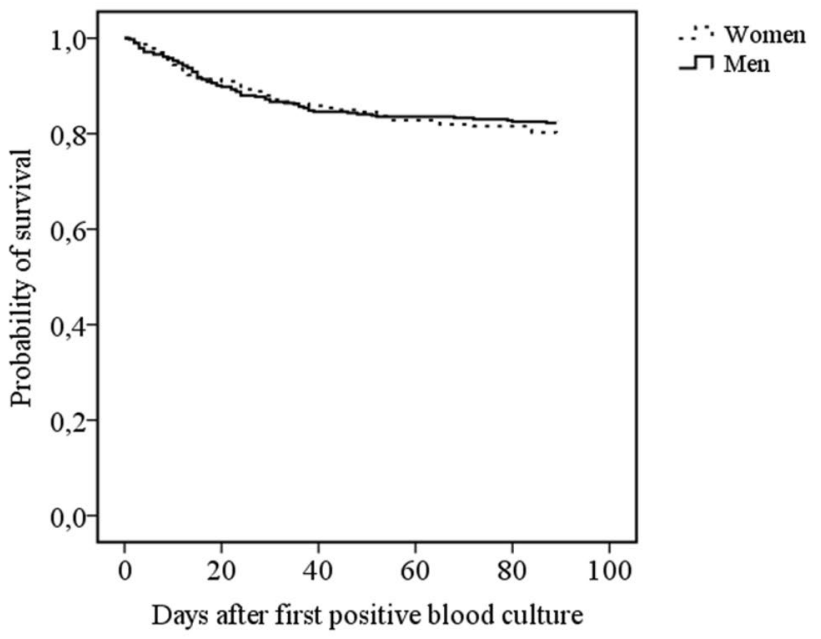

Figure 2

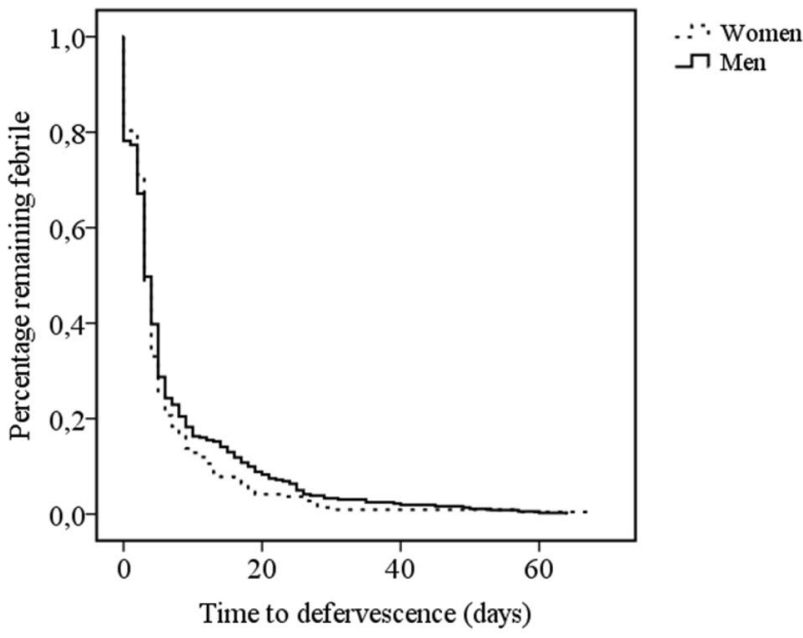

Fig. 1, 2 Kaplan-Meier of 90-days survival and time to defervescence in 617 methicillin-sensitive $S$. aureus bacteremia patients categorized according to sex. Log rank non-significant

studies report female sex as an independent negative prognostic factor in multivariate logistic regression analysis with odd ratios of 1.54-1.59, i.e., closely resembling reported odd ratios of age (1.03), bedridden status on admission (1.54), heart valve disease (1.56) or chronic renal failure (1.92) [12, $15,16]$. In the present study, sex did not connect to 90-day outcome in propensity-score adjusted Cox regression analysis and the hazard ratio of men and women were $0.84 \mathrm{vs}$. 1.0. Moreover, only $1 \%$ of patients presented a SAB relapse within 90 days without any sex difference observed. Previous studies investigating the role of sex in SAB have not documented or reported bacteremia relapse rates $[13,16]$. The extensive diagnostics and eradication of deep infection foci in the present study has most likely reduced the degree of observed SAB relapses.
Previous reports present potential mechanisms for the association of female sex to poor outcome in SAB. Parameters such as age, co-morbidity and $\mathrm{SAB}$ acquisition are factors with well-known prognostic impact in SAB [1-6]. However, sex-related variation in these parameters have been marginal in earlier reports $[13,16]$ and one report concluded that age or co-morbidity did not explain outcome differences between men and women [13]. These observations are in line with the present study observing no variations in age or underlying conditions for men and women. However, we observed less alcoholism and more nosocomial SAB in females, nevertheless, when accounting for these variations in propensity-score adjusted analysis no connection of sex to mortality was observed.

Infectious specialist consultation provides evidence for improved clinical management by accelerating diagnostics, localization and eradication of deep infection foci $[4,6-8$, 27]. ISC was provided irrespective of sex to $94 \%$ of patients and the vast majority ( $84 \%$ ) had formal bedside ISC. The present study observed overall deep infection foci occurrence of $75 \%$ including endocarditis in $15 \%$ and osteomyelitis (or septic arthritis) in $34 \%$ of patients. This exceeds previous studies evaluating the prognostic impact of sex in $\mathrm{SAB}$ with reports of endocarditis in 9-12\% and osteomyelitis in $6-28 \%$ of patients $[13,15,16]$. Comparison of deep infection foci is challenging due to lack of [12] or partial reporting [13-17] of infection foci in previous studies. However, most important, ISC improve outcome $[4,6,27]$ and ISC as a mandatory practice in $\mathrm{SAB}$ is advocated by ever more clinicians [7, 8]. Comparison of ISC guided clinical management is not possible or challenging due to the fact that ISC has been neglected [12-16] or reported incompletely [17] in reports connecting female sex to poor outcome in SAB.

In the present study, standard first-line antibiotic therapies were provided equivalently for men and women and all patients had proper non-delayed antibiotic therapy from the first day of positive blood cultures. This enabled comparison of men and women without the bias from differences in empirical antibiotic therapy. Studies on prognostic impact of sex in SAB have not commented on adjunctive antimicrobial therapies [12-17]. In the present study half of patients received rifampicin or fluoroquinolone adjunctive therapy and rifampicin was provided more often to men compared to women. Adjunctive rifampicin therapy has been associated to a positive prognostic impact among MS-SAB patients with deep infection foci [24]. Corresponding results were achieved also in the present study where $75 \%$ of patients had a deep infection diagnosed. However, the ARREST-study, a recent randomized, double-blind, placebo-controlled trial localized deep infection foci in $40 \%$ of SAB patients and did not observe any positive influence on prognosis due to adjunctive rifampicin therapy although a small significant reduction in bacteriologically and clinically defined disease 
Table 3 Patient characteristics in 116 patients with methicillinsensitive $S$. aureus bacteraemia who deceased within 90-day follow-up

\begin{tabular}{|c|c|c|c|c|}
\hline Patient characteristics & $\begin{array}{l}\text { Deceased women } \\
47(41)\end{array}$ & $\begin{array}{l}\text { Deceased men } \\
69(59)\end{array}$ & OR $(95 \% \mathrm{CI})$ & $p$ value \\
\hline Age $>60$ years & $32(68)$ & $46(67)$ & $0.94(0.43-2.07)$ & NS \\
\hline Nosocomial bacteremia & $31(66)$ & $39(56)$ & $0.67(0.31-1.45)$ & NS \\
\hline Healthy-nonfatal disease ${ }^{a}$ & $20(43)$ & $22(32)$ & $0.63(0.29-1.36)$ & NS \\
\hline \multicolumn{5}{|l|}{ Severity of illness } \\
\hline Severe sepsis ${ }^{b}$ & $9(19)$ & $15(22)$ & $1.17(0.46-2.95)$ & NS \\
\hline PITT scores $\geq 3^{\mathrm{b}}$ & $12(26)$ & $16(23)$ & $0.85(0.36-2.01)$ & NS \\
\hline ICU at bacteremia onset ${ }^{b}$ & $22(27)$ & $27(39)$ & $0.75(0.35-1.59)$ & NS \\
\hline ICU during 7 first days & $16(34)$ & $22(32)$ & $0.91(0.41-2.00)$ & NS \\
\hline \multicolumn{5}{|c|}{ Infectious specialist engagement } \\
\hline Formal consultation & $33(70)$ & $49(71)$ & $1.04(0.46-2.34)$ & NS \\
\hline Informal consultation & $8(17)$ & $10(14)$ & $0.83(0.30-2.28)$ & NS \\
\hline \multicolumn{5}{|l|}{ Echocardiography } \\
\hline Transthoracic & $28(60)$ & $36(52)$ & $0.74(0.35-1.57)$ & NS \\
\hline Transesophageal & $7(15)$ & 13 (19) & $1.33(0.49-3.62)$ & NS \\
\hline Any deep focus & $37(79)$ & $56(81)$ & $1.16(0.46-2.93)$ & NS \\
\hline Pneumonia & $26(55)$ & $41(59)$ & $1.18(0.56-2.50)$ & NS \\
\hline Endocarditis & $10(21)$ & $16(23)$ & $1.12(0.46-2.73)$ & NS \\
\hline \multicolumn{5}{|l|}{ Antibiotic therapy } \\
\hline Staphylococcal penicillin & $24(51)$ & $38(55)$ & $1.18(0.56-2.47)$ & NS \\
\hline Cephalosporin & $16(34)$ & $25(36)$ & $1.10(0.51-2.40)$ & NS \\
\hline Fluoroquinolone $^{\mathrm{c}}$ & $23(49)$ & $27(39)$ & $0.67(0.32-1.42)$ & NS \\
\hline Rifampicin ${ }^{\mathrm{c}, \mathrm{d}}$ & $11(23)$ & $19(28)$ & $1.24(0.53-2.93)$ & NS \\
\hline
\end{tabular}

Patients are stratified according to gender. Values are expressed as $n(\%)$ and odds ratios (ORs) with 95\% confidence intervals (CI) unless otherwise stated

NS non-significant

${ }^{a}$ McCabe's classification [26]

${ }^{\mathrm{b}}$ At blood culture collection

${ }^{\mathrm{c}}$ Adjunctive therapy

${ }^{\mathrm{d}}$ For at least 14 days recurrences was observed [28]. The authors cannot explain why men received more often rifampicin adjunctive therapy in the present study. However, despite inequality in distribution of adjunctive rifampicin therapy between men and women, the propensity-score adjusted Cox proportional analysis, accounting for all prognostic factors, observed no independent prognostic impact of sex.

Reports have proposed that gender dimorphism [18] and female sex steroids [19] may affect immune response with subsequent potential effect on outcome. However, the mean age for female patients in the present study was 58 years (and only $12 \%$ of the whole patient coho 0rt were women under the age of 50 years), i.e., most women were presumably of postmenopausal age. However, although postmenopausal age may reduce hormonal influence on immuno-status there might be some relevant differences in sex hormone or steroid levels between men and women. The present study did not record sex hormone or steroid levels. However, we looked for sex differences in laboratory tests including white blood cell count, parameters for coagulation, basic immunoglobulins, complement factors, teichoic acid antibody and antistaphylolysin and could not observe any sex-related differences.

Previous reports on sepsis management have presented alarming results on inequality of clinical management for severely ill female patients including delayed empiric antimicrobial initiation [21] and less access to mechanical ventilation, hemodialysis care [22] or life-supporting treatments [23]. A recent thorough report on sex-related outcome in SAB [13] and a subsequent editorial commentary [26] concluded that non-biological factors, including potential sex-related treatment inequalities, may explain outcome differences between men and women and argued that for the present time female sex is to be viewed as a potential prognostic parameter requiring triage and risk stratification. We did not observe any differences in clinical management, with the exception of adjunctive rifampicin therapy, between men and women. 
Table 4 Propensity-score adjusted Cox proportional regression model analysis for 90-day mortality among 605 patients with methicillinsensitive $S$. aureus bacteremia

\begin{tabular}{lll}
\hline Prognostic parameters & $\begin{array}{l}\text { PS-adjusted multivariate } \\
\text { HR }(95 \% \mathrm{CI})\end{array}$ & $p$ value \\
\hline Men & $0.84(0.56-1.27)$ & NS \\
Women & 1.0 & - \\
Age $>60$ years & $2.21(1.41-3.45)$ & $<0.01$ \\
Healthy-nonfatal disease $^{\mathrm{a}}$ & $0.23(0.15-0.35)$ & $<0.001$ \\
Intensive care unit $^{\mathrm{b}}$ & $1.60(1.04-2.46)$ & $<0.05$ \\
Formal ISC $^{\mathrm{c}}$ & $0.58(0.36-0.93)$ & $<0.05$ \\
Endocarditis diagnosis & $2.51(1.56-4.04)$ & $<0.001$ \\
Pneumonia diagnosis $^{\mathrm{d}}$ & $2.42(1.60-3.66)$ & $<0.001$ \\
Rifampicin therapy $^{\mathrm{d}}$ & $0.29(0.18-0.47)$ & $<0.001$ \\
\hline
\end{tabular}

Hazards ratio (HR) and 95\% confidence intervals (95\% CI) are presented. Patients deceased within three first days $(N=12)$ have been excluded

${ }^{a}$ McCabe's classification [26]

${ }^{\mathrm{b}}$ At blood culture collection time

${ }^{\mathrm{C}}$ Infectious specialist consultation

${ }^{\mathrm{d}}$ Adjunctive therapy $\geq 14$ days

However, the majority of patients had ISC which is known to improve disease progression and prognosis in SAB [4, $6-8,27]$. Hence, it may be speculated that ISC balances potential sex-related differences in SAB management. However, such conclusions cannot be drawn due to the lack of control groups (without ISC) in the present study.

There are weaknesses in the present study that have to be accounted for when interpreting results. First, differences in patient groups may bias analyses of outcome. However, propensity-score adjustment and data categorization may reduce potential bias [29]. The results were achieved by propensity-score adjustment, hence, author's view that the results are robust and the risk for statistical bias low. Second, data were collected in years 1999-2002 and 2006-2007 and the question of whether this data is valid to current clinical practice may be raised. However, although clinical management of SAB is continuously developed as new research is published, there are fundamental elements of SAB treatment that have remained unchanged over the years such as the importance of nondelayed onset of proper antibiotic treatment and identification and eradication of infection foci. ISC The authors view that the high presence of ISC in the present study has ensured recording of relevant clinical patient information and hence enabled high standard clinical management of $\mathrm{SAB}$. Hence, the authors view that patient data of the present study is not outdated.

In conclusion, patient characteristics, clinical management, ISC guidance, bacteremia relapse, and outcome do not differ in men and women with MS-SAB.
Funding The study has been supported by grants from The Medical Society of Finland, the Perkléns Foundation and the Maud Kuistila Foundation. The funders had no role in study design, data collection and analysis, decision to publish, or preparation of the manuscript.

\section{Compliance with ethical standards}

Conflict of interest The authors declare that they have no conflict of interest. On behalf of all authors, the corresponding author states that there is no conflict of interest.

Ethics statement The trial was approved by the institutional review board of Helsinki University Central Hospital and the ethical committee of Helsinki University Central Hospital.

\section{References}

1. Braquet $\mathrm{P}$, Alla F, Cornu C, et al. Factors associated with 12 week case-fatality in Staphylococcus aureus bacteraemia: a prospective cohort study. Clin Microbiol Infect. 2016;22:948.e1-948.e7.

2. Kang CI, Song JH, Ko KS, et al. Asian Network for Surveillance of Resistant Pathogens Study Group. Clinical features and outcome of Staphylococcus aureus infection in elderly versus younger adult patients. Int J Infect Dis. 2011;15:58-62.

3. Ammerlaan H, Seifert H, Harbarth S, et al. Adequacy of antimicrobial treatment and outcome 334 of Staphylococcus aureus bacteremia in 9 Western European countries. Clin Infect Dis. 2009;49:997-1005.

4. Rieg S, Peyerl-Hoffmann G, de With K, et al. Mortality of S. aureus bacteremia and infectious diseases specialist consultation-a study of 521 patients in Germany. J Infect. 2009;59:232-9.

5. Cosgrove S, Sakoulas G, Perencevich E, et al. Comparison of mortality associated with methicillin resistant and methicillinsusceptible Staphylococcus aureus bacteremia: a meta-analysis. Clin Infect Dis. 2003;36:53-9.

6. Honda $\mathrm{H}$, Krauss $\mathrm{M}$, Jones J, et al. The value of infectious diseases consultation in Staphylococcus aureus bacteremia. Am J Med. 2010;123:631-7.

7. Saunderson R, Gouliouris T, Nickerson E, et al. Impact of routine bedside infectious disease consultation on clinical management and outcome of Staphylococcus aureus bacteraemia in adults. Clin Microbiol Infect. 2015;21:779-85.

8. Martin L, Harris M, Brooks A, et al. Management and outcomes in patients with Staphylococcus aureus bacteremia after implementation of mandatory infectious diseases consult: a before/after study. BMC Infect Dis. 2015; 15:568.

9. Adrie C, Azoulay E, Francais A, et al. Influence of gender on the outcome of severe sepsis: a reappraisal. Chest. 2007;132:1786-93.

10. Leibovici L, Paul M, Weinberger M, et al. Excess mortality in women with hospital-acquired bloodstream infection. Am J Med. 2001;111:120-5.

11. Madsen T, Simmons J, Choo E, et al. The disparity study: Do gender differences exist in surviving sepsis campaign resuscitation bundle completion, completion of individual bundle elements, or sepsis mortality? J Crit Care. 2014;29:473 7-11.

12. Mejer N, Westh H, Schønheyder HC, et al. Stable incidence and continued improvement in the short term mortality of Staphylococcus aureus bacteremia between 1995-2008. BMC Infect Dis. 2012;12:260.

13. Smit J, Lopez-Cortes LE, Kaasch AJ, et al. Gender differences in the outcome of community-acquired Staphylococcus aureus 
bacteraemia: a historical population-based cohort study. Clin Microbiol Infect. 2017;23:27-32.

14. Lamagni T, Potz N, Powell D, et al. Mortality in patients with meticillin-resistant Staphylococcus aureus bacteraemia, England 2004-2005. J Hosp Infect. 2011;77:16-20.

15. Allard C, Carignan A, Bergevin M, et al. Secular changes in incidence and mortality associated with Staphylococcus aureus bacteraemia in Quebec, Canada, 1991-2005. Clin Microbiol Infect. 2008;14:421-8.

16. Mansur N, Hazzan R, Paul M, et al. Does sex affect 30-day mortality in Staphylococcus aureus bacteremia. Gender Med. 2012;9:463-70.

17. Yahav D, Yassin S, Shaked H, et al. Risk factors for long-term mortality of Staphylococcus aureus bacteremia. Eur J Clin Microbiol Infect Dis. 2016;35:785-90.

18. Humphreys H, Fitzpatrick F, Harvey BJ. Gender differences in rates of carriage and bloodstream infection caused by methicillinresistant Staphylococcus aureus: are they real, do they matter and why? Clin Infect Dis. 2015;61:1708-14.

19. Angele MK, Pratschke S, Hubbard WJ, et al. Gender difference in sepsis: cardiovascular and immunological aspects. Virulence. 2014;5:12-9.

20. Schroder W, Sommer H, Gladstone BP, et al. Gender differences in antibiotic prescribing in the community: a systematic review and meta-analysis. J Antimicrob Chemother. 2016;71:1800-6.

21. Madsen TE, Napoli AM. The disparity-ii study: delays to antibiotic administration in women with severe sepsis or septic shock. Acad Emerg Med. 2014;21:1499-502.
22. Pietropaoli AP, Glance LG, Oakes D, et al. Gender differences in mortality in patients with severe sepsis or septic shock. Gender Med. 2010;7:422-37.

23. Raine R, Goldfrad C, Rowan K, et al. Influence of patient gender on admission to intensive care. J Epidemiol Community Health. 2002;56:418-23.

24. Ruotsalainen E, Järvinen A, Koivula I, et al. Levofloxacin does not decrease mortality in Staphylococcus aureus bacteraemia when added to the standard treatment: a prospective and randomized clinical trial of 381 patients. J Intern Med. 2006;259:179-90.

25. Tacconelli E, Foschi F. Does gender affect the outcome of community-acquired Staphylococcus aureus bacteraemia? Clin Microbiol Infect. 2017;23:23-5.

26. McCabe WR, Jackson GG. Gram-negative bacteremia: I. Etiology and ecology. Arch Intern Med. 1962;110:847-55.

27. Forsblom E, Ruotsalainen E, Ollgren J, et al. Telephone consultation cannot replace bedside infectious disease consultation in the management of Staphylococcus aureus bacteraemia. Clin Infect Dis. 2012;56:527-35.

28. Thwaites GE, Scarborough M, Szubert A, et al. Adjunctive rifampicin for Staphylococcus aureus bacteraemia (ARREST): a multicentre, randomised, double-blind, placebo-controlled trial. Lancet. 2018;17:668-78.

29. Rosenbaum PR, Rubin DB. The central role of the propensity score in observational studies for causal effects. Biometrika. 1983;70:41-55. 